\title{
On the Determination of Eigenmodes and Eigenfrequecies of Two- Junctioned Carbon Nanotubes under the Influence of Microscopic Defects
}

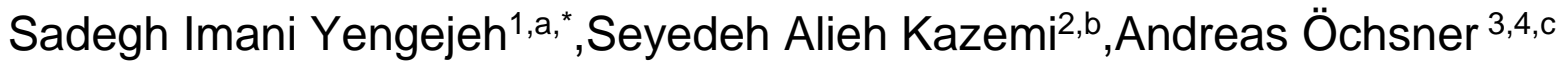 \\ ${ }^{1}$ School of Engineering, Griffith University, Gold Coast Campus, \\ Southport 4222, Australia \\ ${ }^{2}$ Department of Mechanical Engineering, The University of Birjand, \\ Birjand, Iran \\ ${ }^{3}$ School of Engineering, Griffith University, Gold Coast Campus, \\ Southport 4222, Australia \\ ${ }^{4}$ School of Engineering, The University of Newcastle, Callaghan \\ New South Wales 2308, Australia \\ almani.sd@gmail.com ('Corresponding author), bSa.kazemi83@gmail.com, \\ cAndreas.Oechsner@gmail.com
}

Keywords: nanostructures; defects; mechanical properties

\begin{abstract}
Connected CNTs were simulated and the effect of impurities on the frequency of these specific nano-structures was analyzed. For this purpose, the three most likely microscopic imperfections, i.e., doping with Si atoms, vacancy and perturbations to the pure models were simulated. Lastly, the vibrational behavior of imperfect hybrids was evaluated and compared with the behavior of the pure ones. It was pointed out that deficiencies in the structure of hybrids reduces the frequency and as a result, lowers the vibrational stability of the CNTs.
\end{abstract}

\section{Introduction}

Carbon nanotubes (CNTs) are components of nanoscale dimensions that present outstanding physical, mechanical and electrical properties [1]. These particular behavior have made them potentially valuable for many applications in nanoengineering, electronics and other fields of materials science. Consequently, numerous investigations have been conducted to predict the behavior of these nano-structures in the recent two decades. These investigations can be separated into two groups of experimental and computational approaches. Molecular dynamics and continuum mechanics methods have been the most predominant computational approaches to evaluate the behavior, e.g. the vibrational behavior, of CNTs. In the following, the outcomes of several studies on the evaluation of the nanotubes vibrational behavior are presented.

In 2009, Hashemnia et al. [2] studied the vibrational properties of two kinds of single layered graphene sheets and single-walled CNTs. They carried out the simulations for different types of zigzag and armchair nanotubes and graphene sheets with various boundary conditions. They obtained the frequency by means of a molecular structural mechanics approach where the nano-structures were considered as space frames. Based on their findings, the frequency 
drops as aspect ratio increases. Thus, it is preferred to use CNTs and graphene sheets with lower aspect ratios for dynamic applications to prevent resonance and dynamic damage. They also pointed out that the frequency of CNTs is larger than that of graphene sheets. Later in 2010, Filiz and Aydugdu [3] studied the axial vibration of carbon nanotube two-junctions, applying a nonlocal rod theory. They used the nonlocal constitutive equations of Eringen in their formulations. They thoroughly investigated the influence of nonlocality, length of the CNTs and lengths of each segment for each considered problem. It was finally concluded that by joining CNTs good vibrational behavior are obtained by suitable selection of parameters. Afterwards in 2011, a comprehensive numerical study on the free and forced vibration of single-walled CNTs was presented by Arghavan and Singh [4]. They developed a simple approach such that the proximity of the mathematical model to the actual atomic structure of the CNT is considerably retained. They obtained the natural frequencies and corresponding mode shapes of different types of CNTs with different boundary conditions. Outcomes concerning axial, bending, and torsional modes of vibration were reported. Recently in 2012, Seyyed Fakhrabadi et al. [5] studied the vibrational behavior of CNT hybrids with different geometries and boundary conditions. The common molecular mechanics approach was applied to the modal analysis of the mentioned nanoparticles and they obtained the natural frequencies of CNTs and their corresponding mode shapes. They pointed out that the tighter boundaries led to higher natural frequencies. Furthermore, the larger diameters and shorter lengths resulted in higher natural frequencies. As reviewed above, the vibrational properties of the CNTs were investigated in detail but junctioned CNTs were only rarely addressed. Since carbon nanotube intramolecular junctions are expected to have a high potential for application in miniature electronic devices [6], a more complete characterisation of their physical properties may facilitate their application. The results of the current investigation allow a genuine estimate of the vibrational behavior of nanostructures in a closer form to those found in reality. The aim of the current research is to continue the previous investigations $[7,8,9,10]$ and study the behavior of defective two-junction CNTs. These imperfections are carbon vacancy, perturbation and Si-doping. Previous investigations examined the vibrational response of pure hetero-junctions without imperfection and curved homogenous CNT, respectively.

\section{Methodology}

\section{Geometric definition}

CNTs are hollow cylinder shaped structures. The geometry of a CNT is defined by the chiral vector $\boldsymbol{C}_{h}$ and the chiral angle $\theta$. The chiral vector is represented by two unit vectors $\boldsymbol{a}_{1}$ and $\boldsymbol{a}_{2}$ and two integers $m$ and $n$ as it is shown by the following equation [11]:

$$
\boldsymbol{C}_{h}=n \boldsymbol{a}_{1}+m \boldsymbol{a}_{2}
$$

The simple structure of CNTs can be defined according to the chiral vector or angle, as shown in Fig. 1. 


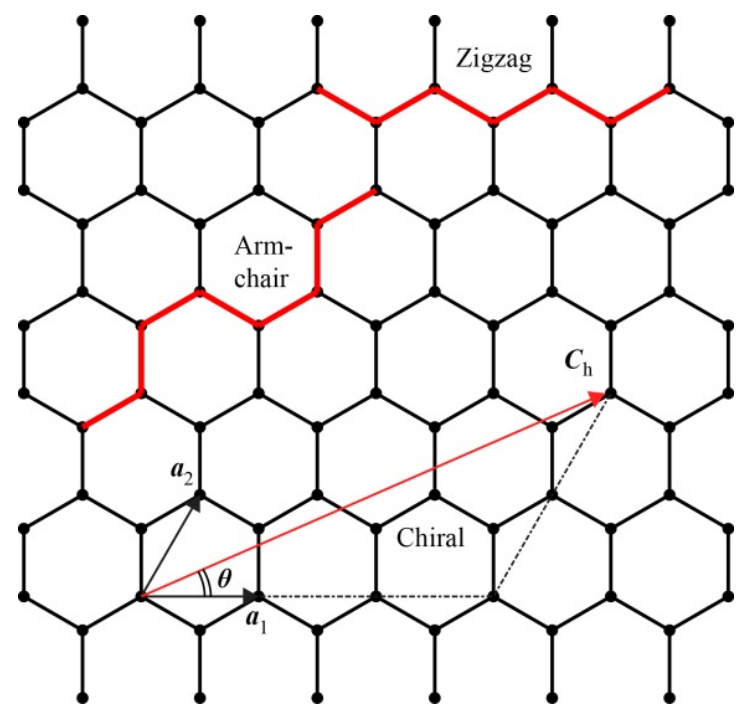

Figure 1. Schematic illustration showing armchair, zigzag and chiral CNTs

A two-junction CNT, is constructed by joining two nanotubes through the introduction of a 57 pair defect into the linking region, as shown in Figs. 2-3.

(a)

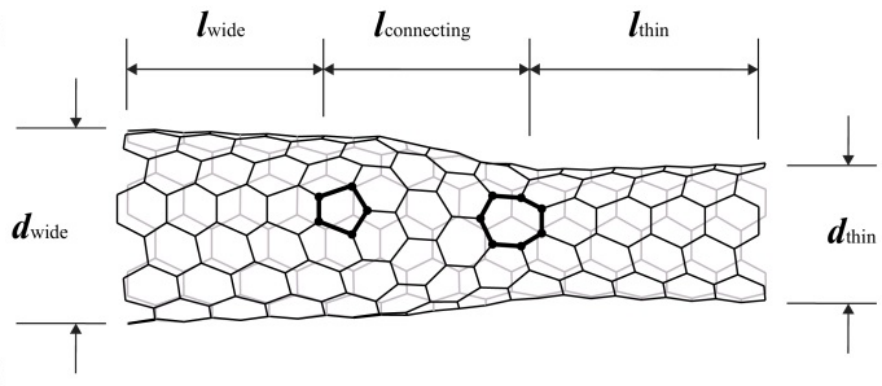

(b)

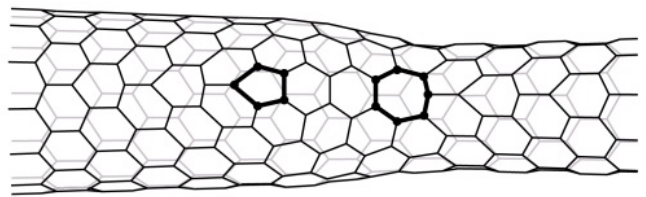

(c)

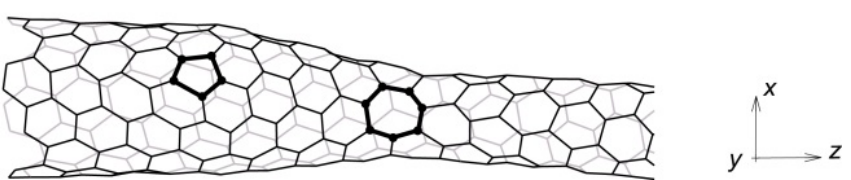

Figure 2. Front view of (a) $(5,5)-(7,7)$, (b) $(9,0)-(11,0)$ and (c) $(5,2)-(8,4)$ straight hetero-junction CNTs. 


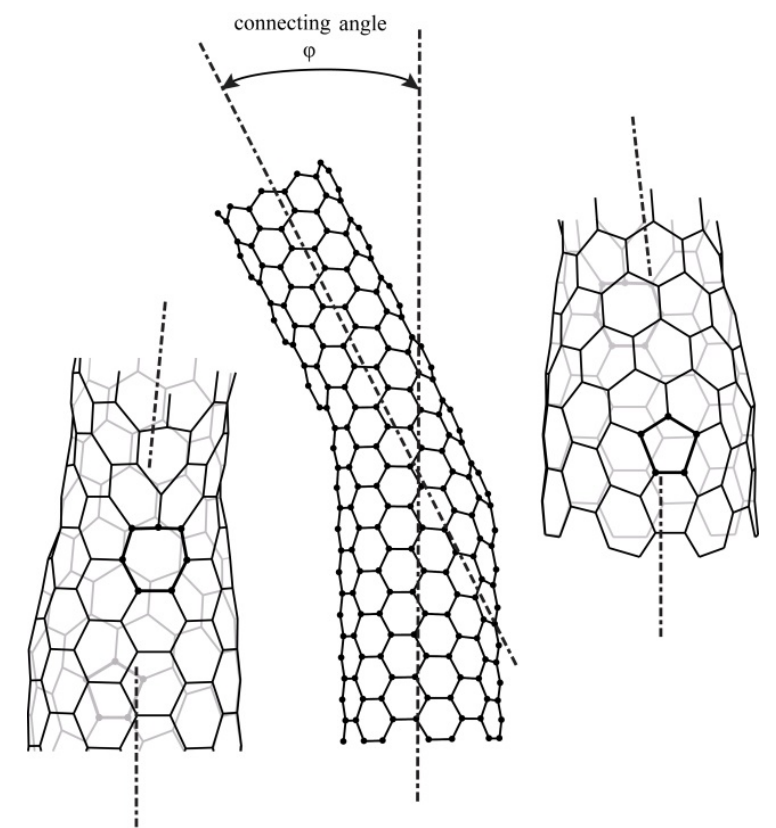

Figure 3. (7,7)-(9,0) kink hetero-junction CNT

These specific types of nano-structures are separated based on the foundations of their two basic CNTs. For instance, a CNT hybrid made by linking a $(5,5)$ armchair CNT and a $(7,7)$ armchair tube is referred to as a $(5,5)-(7,7)$ hetero-junction CNT [12]. Figure 2 illustrates some models of straight hetero-junctions with different types of fundamental homogenous CNTs.

\section{Simulation of defective CNT configurations}

In this paper, the three most likely microscopic modifications, i.e., Si-doping, vacancy and perturbation were introduced to the ideal hetero-junctions. All the basic calculations were performed by MATLAB and the acquired defective hetero-junctions under cantilever boundary conditions were generated by means of the finite element software MSC.Marc to evaluate their vibrational response in comparison with the results of ideal two-junctioned nanotubes.

\section{Vibrational behavior}

The natural frequency is the basic frequency of a vibrating system at which the system oscillates at greater amplitude due to the existence of the resonance phenomenon. The first natural frequency of an ideal Euler-Bernoulli beam element under fixed-free boundary conditions is well-defined by the following equation [13]:

$f=(3.5156 / 2 \pi) \sqrt{E I / \bar{m} L^{4}}$

where $E, I, \bar{m}$ and $L$ are the Young's modulus, the second moment of area, the mass per unit length and the length of the CNT, respectively. In the finite element simulations, the Young's modulus of CNTs was calculated by the following equations: 
$\sigma=$ stress $=P / A=\frac{\text { reaction force }}{\text { cross }- \text { sectional area }}$

$\varepsilon=$ strain $=\Delta L / L=\frac{\text { displacement B. C. }}{\text { length of CNT }}$

$E=$ Young's modulus $=\sigma / \varepsilon$

As the configuration of CNTs is frequently offered by a hollow tube, Eq. (6) can be used to obtain the structure's second moment of area for ideal nanotubes as:

$I=\pi\left[(d+t)^{4}-(d-t)^{4}\right] / 64$

where $t$ is the thickness of the tube's shell and $d$ represents the diameter of the nanotube. The value of the mass per unit length can be obtained from the following equation:

$\bar{m}=m_{\text {total }} / l_{\text {total }}$

where $m_{\text {total }}$ is the total mass of the CNT carbon atoms and $l_{\text {total }}$ is the total length of the CNTs.

\section{Results and discussion}

Vibrational behavior of perfect hetero-junctions

The vibrational behavior of straight and kink two-junctions is inspected by investigating their first natural frequency computationally applying a finite element method. For the FE technique, first, straight and bend nanotube hybrids were computer-generated. After that, by introducing the fixed-free boundary condition, the vibrational behavior of the heterojunctions was investigated. Finally, the computations presented the first natural frequency of these specific types of nano-particles. Figure 4 shows the first six natural frequencies of perfect straight and kink hetero-junction CNTs. 
(a)

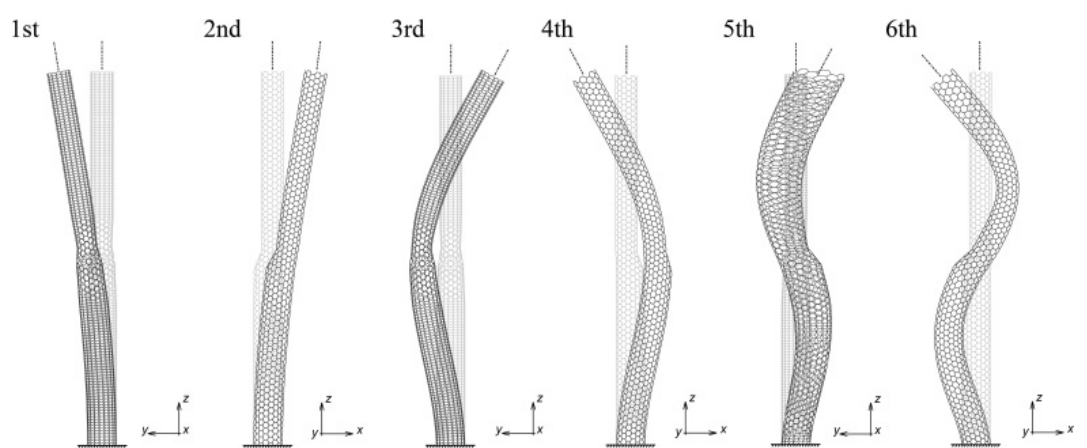

(b)
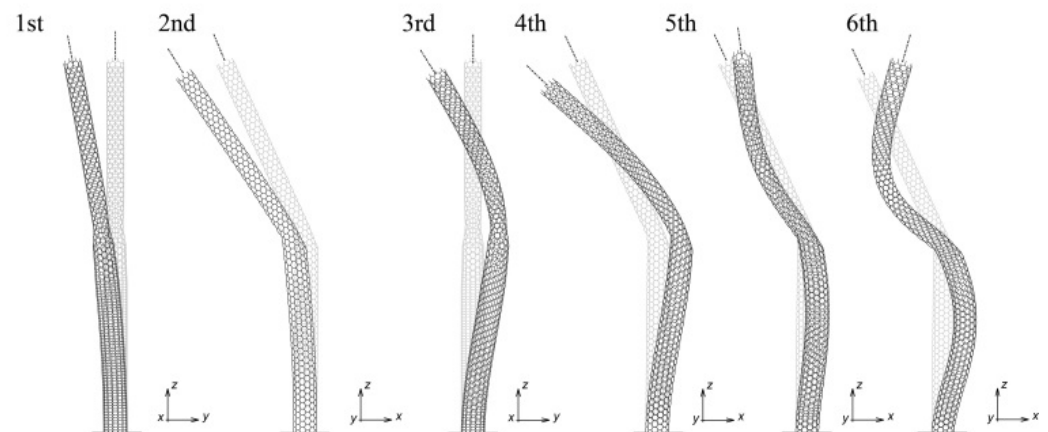

Figure 4. First six eigenmodes of $\overline{(\mathbf{a})}$ straight and (b) kink CNT hybrids

Vibrational properties of defective hetero-junctions

Vibrational behavior of Si-doped hetero-junctions

First, the two-junctioned tubes were defected by replacing 2, 4, 6, 8 and 10\% of the total number of carbon atoms in the ideal structure with silicon atoms. The outcomes illustrated in Fig. 5 indicate that an increase in the percentage of doping leads to a gradual reduction of the natural frequency in both circumstances of straight and bend nanotube hybrids. 
(a)

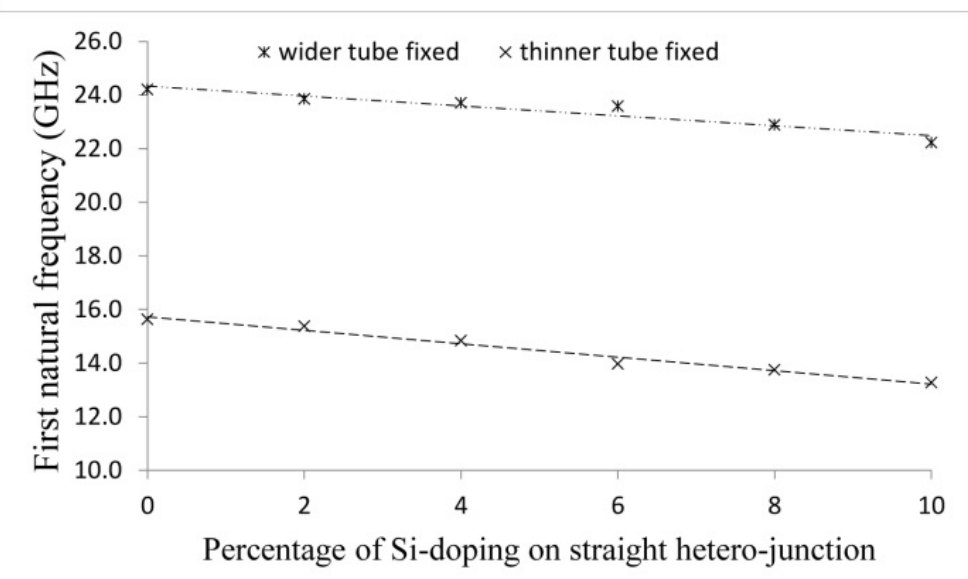

(b)

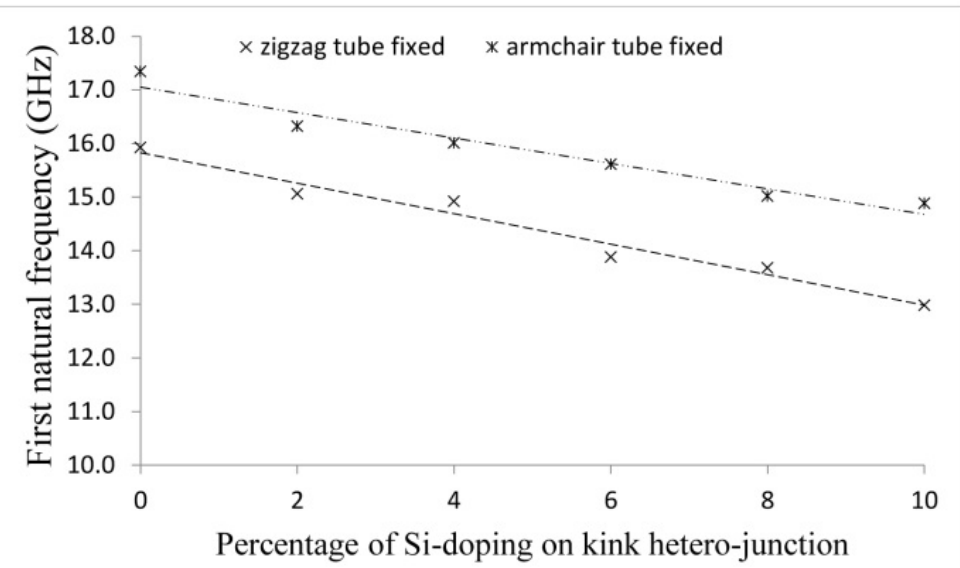

Fig. 5. Change in natural frequency caused by Si-doping of (a) $(3,3)-(5,5)$ straight hetero-junction and (b) $(5,0)-(7,0)$ kink hetero-junction CNT

Vibrational behavior of hetero-junctions with carbon vacancy

For the second case, the deficiency was introduced by creating vacancies in the structure of defect-free hetero-junctions. For this determination, 1, 2, 3, 4 and $5 \%$ of atoms were randomly chosen and eliminated from the configuration of the tubes. The results in Fig. 6 show that vacancies considerably decline the natural frequency of these specific two junctioned CNTs. 
(a)

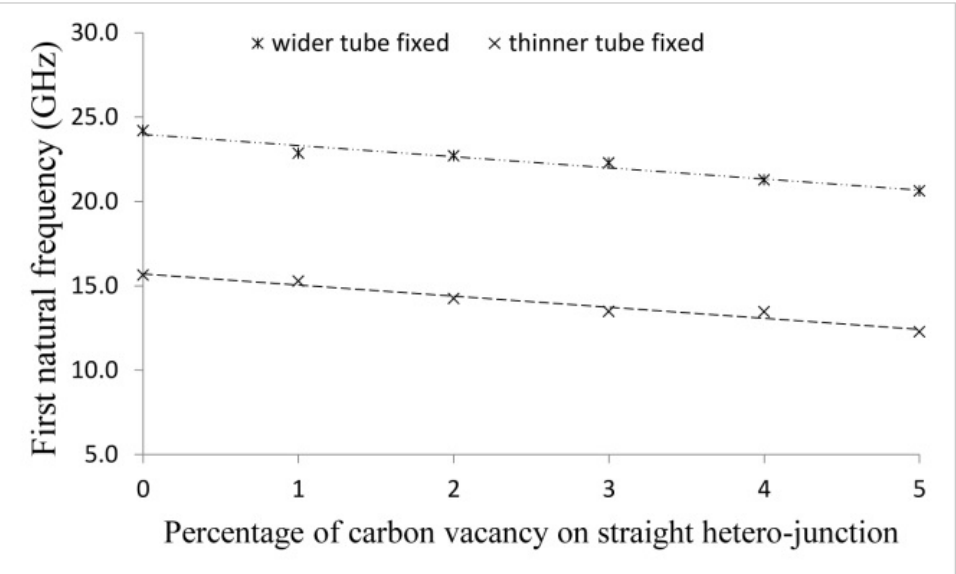

(b)

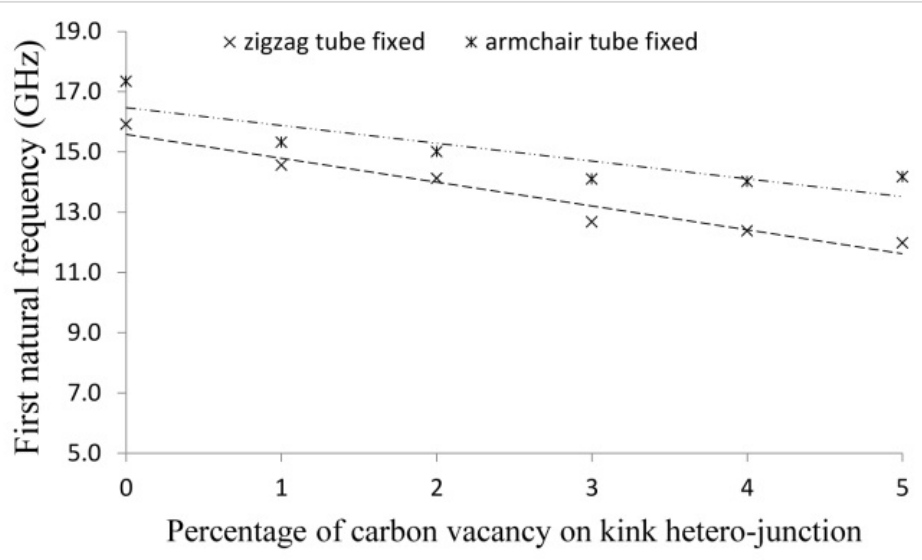

Fig. 6. Change in natural frequency caused by carbon vacancies in (a) $(3,3)-(5,5)$ straight heterojunction and (b) $(5,0)-(7,0)$ kink hetero-junction CNT

\section{Vibrational behavior of perturbed hetero-junctions}

The ideal structures were perturbed by 5, 10, 15, 20, 25\%. The findings in Fig. 7 show that perturbation in the structure of two-junctions slightly reduces their natural frequency. 
(a)

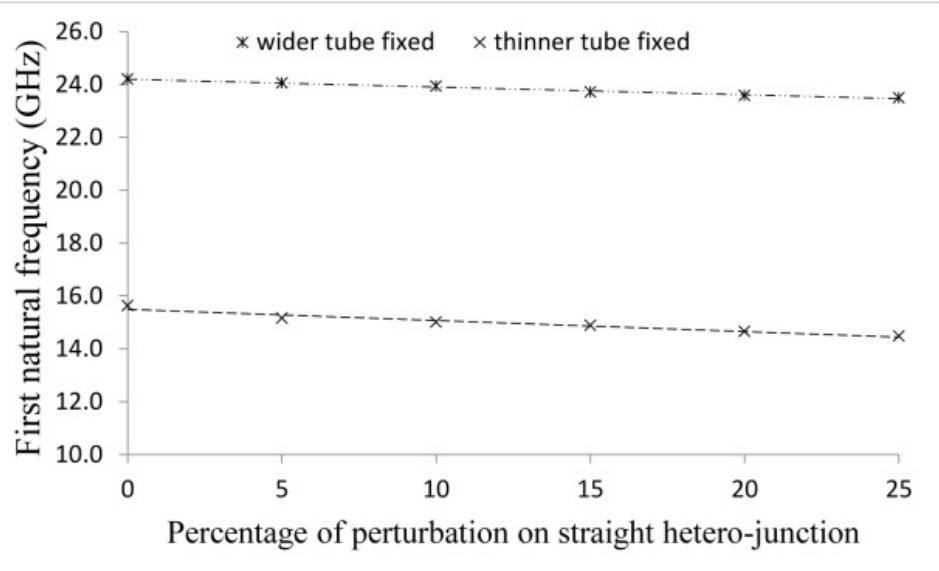

(b)

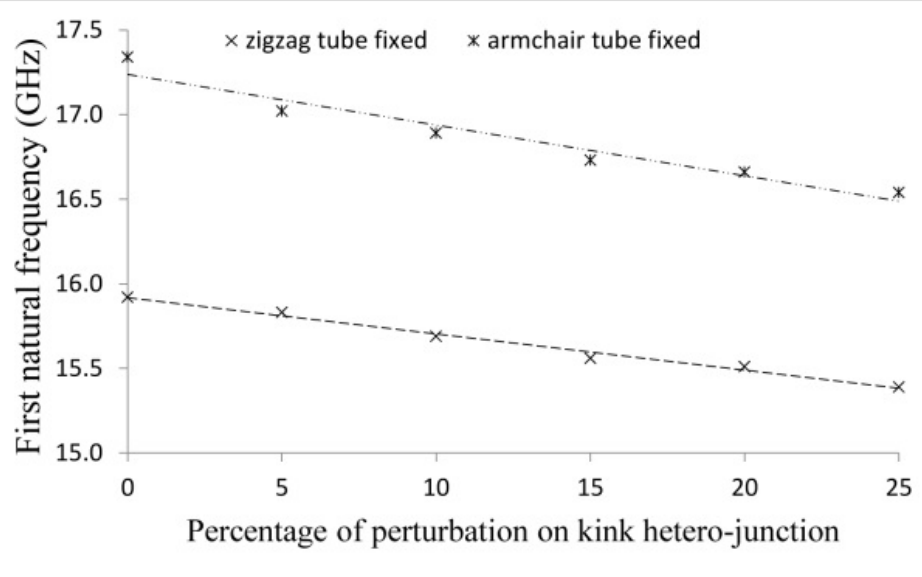

Fig. 7. Change in natural frequency caused by perturbation of $(\mathbf{a})(3,3)-(5,5)$ straight hetero-junction and (b) $(5,0)-(7,0)$ kink hetero-junction CNT

\section{Conclusions}

In the current study, two-junction CNTs were computer-generated in their ideal arrangement. Then three types of microscopic defects were introduced to the two-junctioned CNTs. It was indicated that the existence of imperfections in the arrangement of hetero-junctions leads to a lower natural frequency. The vibrational stability of hetero-junction CNTs also drops by a growth of the percentage of imperfections. Finally, it should be mentioned that other mechanical properties of atomically reformed two-junctions, e.g. the elastic properties, could be explored in the future studies.

\section{References}

[1] C.W.S. To, Bending and shear moduli of single-walled carbon nanotubes, Finite Elem. Anal. Des. 42 (2006) 404-413. 
[2] K. Hashemnia, M. Farid, R. Vatankhah, Vibrational analysis of carbon nanotubes and graphene sheets using molecular structural mechanics approach. Comp. Mater. Sci. 47 (2009) 79-85.

[3] S. Filiz and M. Aydugdu, Axial vibration of carbon nanotube hetero-junctions using nonlocal elasticity. Comp. Mater. Sci. 49 (2010) 619-627.

[4] S. Arghavan and A.V. Singh, On the vibrations of single-walled carbon nanotubes. J. Sound Vib. 330 (2011) 3102-3122.

[5] M.M. Seyyed Fakhrabadi, A. Amini, A. Rastgoo, Vibrational properties of two and three junctioned carbon nanotubes. Comp. Mater. Sci. 65 (2012) 411-425.

[6] Z. Yao, H.W.C. Postma, L. Balents, C. Dekker, Carbon nanotube intramolecular junctions. Nature 402 (1999) 273-276.

[7] S. Imani Yengejeh, M. Akbar Zadeh, A. Öchsner, Numerical modeling of eigenmodes and eigenfrequencies of hetero-junction carbon nanotubes with pentagon-heptagon pair defects. Comp. Mater. Sci. 92 (2014) 76-83.

[8] S. Imani Yengejeh, J.M.P.Q. Delgado, A.G.B. de Lima, A. Öchsner, Numerical Simulation of the Vibration Behavior of Curved Carbon Nanotubes. Advances Mater Sci Eng. http://dx.doi.org/10.1155/2014/815340.

[9] A. Ghavamian and A. Öchsner. Numerical modeling of eigenmodes and eigenfrequencies of single-and multi-walled carbon nanotubes under the influence of atomic defects. Comp. Mater. Sci. 72 (2013) 42-48.

[10] S. Imani Yengejeh, S.A. Kazemi, A. Öchsner, Advances in mechanical analysis of structurally and atomically modified carbon nanotubes and degenerated nanostructures: A review. Composites Part B: Engineering 86 (2016) 95-107

[11] M.S. Dresselhaus, G. Dresselhaus, R. Saito, Physics of carbon nanotubes. Carbon 33 (1995) 883-891.

[12] M. Li, Z. Kang, P. Yang, X. Meng, Y. Lu, Molecular dynamics study on buckling of single-wall carbon nanotube-based intramolecular junctions and influence factors. Comp. Mater. Sci. 67 (2013) 390-396.

[13] Irvine T: Application of the Newton-Raphson Method to Vibration Problems. Vib. data Pub 1999. 